\title{
Introdução alimentar precoce em uma coorte de prematuros nascidos em maternidade de alto risco
}

\section{Early food introduction in premature}

\author{
João Ronaldo Silva Monteiro ${ }^{1}$, Micaely Cristina dos Santos Tenório ${ }^{1}$, Carolina Santos Mello², \\ Alane Cabral Menezes de Oliveira ${ }^{{ }^{*}}$
}

\begin{abstract}
RESUMO
O objetivo deste artigo é investigar a frequência do aleitamento materno exclusivo (AME) de prematuros ao longo dos 6 primeiros meses de vida, bem como os alimentos mais frequentemente introduzidos às crianças nesse período. Trata-se de estudo de coorte com recém-nascidos prematuros nascidos em uma maternidade de alto risco de Maceió, Alagoas, acompanhados por meio de ligações telefônicas realizadas a cada 30, 60, 90,120,150 e 180 dias após o anscimento para avaliação da duração do aleitamento materno, do período em que ocorreu a introdução de outros alimentos e quais alimentos foram introduzidos de forma precoce. A coorte incluiu um total de 132 prematuros. Foi observada uma redução de $67,77 \%$ da frequência de AME, com uma prevalência ao final do estudo de $32,23 \%$. Quando detalhados por período de acompanhamento, as prevalência de AME foram de: $91,66 \%$ aos 30 dias, $81,81 \%$ aos 60 dias, $67,68 \%$ aos 90 dias, $58,67 \%$ aos 120 dias, $46,28 \%$ aos 150 dias e de $32,23 \%$ aos 180 dias. Nos prematuros em aleitamento materno misto (AMM), as fórmulas infantis foram os primeiros alimentos introduzidos de forma precoce, seguido do leite de vaca e farináceos.
\end{abstract}

Palavras-chave: Aleitamento materno; Introdução alimentar precoce; Prematuridade.

\section{ABSTRACT}

The aim of this article is to investigate the frequency of exclusive breastfeeding (EBF) of preterm infants during the first 6 months, as well as the foods most frequently introduced during this period. This is a cohort study with premature newborns born in a high-risk maternity hospital in Maceió, Alagoas, followed by telephone calls made every $30,60,90,120,150$ and 180 days to assess the duration of the period. breastfeeding, the period in which other foods were introduced and which foods were introduced early. The cohort included a total of 132 preterm infants. $67.77 \%$ reduction in EBF frequency was observed, with a prevalence at the end of the study of $32.23 \%$. When detailed by follow-up period, the prevalence of EBF were: $91.66 \%$ at 30 days, $81.81 \%$ at 60 days, $67.68 \%$ at 90 days, $58.67 \%$ at 120 days, $46.28 \%$ at 150 days and $32.23 \%$ at 180 days. In preterm infants on mixed breastfeeding (MB), infant formulas were the first foods introduced early, followed by cow's milk and flours.

Keywords: Breastfeeding; Early food introduction; Prematurity.

\footnotetext{
${ }^{1}$ Universidade Federal de Alagoas, Faculdade de Nutrição. Maceió, Alagoas, Brasil.

${ }^{2}$ Universidade Federal da Bahia, Escola de Nutrição. Salvador, Bahia, Brasil.

*E-mail: alanecabral@gmail.com
} 


\section{INTRODUÇÃO}

É bem conhecido na literatura que o aleitamento materno exclusivo (AME), aquele em que há oferta exclusiva de leite materno sem acréscimo de outros alimentos (líquidos ou sólidos) até os 6 primeiros meses de vida, agrega diversos benefícios à saúde da criança, comoo fornecimento de fatores de proteção imunológica, melhor desenvolvimento neuro-cognitivo, fortalecimento do vínculo mãe-filho, suprimento adequado das necessidades nutricionais e redução da morbimortalidade infantil (LANCET, 2016; BRASIL, 2015; KOH, 2017). Estes benefícios se estendem a melhor prognóstico, menor tempo de internação e menores taxas de sepse tardia quando se trata de recém-nascidos prematuros ( $<37$ semanas de gestação) (WILSON et al., 2018), onde evidências mostram que os primeiros mil dias de vida podem repercutir sobre a saúde nas fases subsequentes da vida, determinando o risco de desenvolvimento de doenças crônicas não transmissíveis (DCNT), como a obesidade (D’' AURIA et al., 2020).

A introdução da alimentação complementar deve ser feita a partir dos 6 meses completos de vida, devendo ser realizada de forma a complementar a oferta do leite materno (D' AURIA et al., 2020). Nesta fase se reconhece que o leite materno não é mais suficiente para atender as demandas nutricionais e metabólicas da criança, que já apresentam um maior desenvolvimento neuromuscular que implica numa maior maturidade dos reflexos de mastigação e deglutição, assim como uma maior maturidade fisiológica dos processos de digestão e absorção, denotando um maior preparo da criança em receber novos alimentos (BRASIL, 2015; D' AURIA et al., 2020; WERE, LIFSCHITZ, 2018). Se tratando de prematuros deve-se existir uma atenção com a introdução da alimentação complementar tendo em vista as dificuldades relacionadas a imaturidade fisiológica destes, sendo, em alguns casos adotada a idade corrigida para introdução alimentar de forma a respeitar o tempo necessário para que o lactente desenvolva maturidade fisiológica suficiente para receber novos alimentos (LIOTTO, 2020; BRAID et al., 2015).

A introdução de outros tipos de leite ou fórmulas industriais à base de leite de vaca configura o aleitamento materno misto (AMM) ou parcial (BRASIL, 2015). A prescrição de fórmulas infantis deve ser realizada conforme necessidades individuais, em casos de contraindicação da amamentação ou quando a produção do leite materno não estiver em quantidades suficientes para atender a demanda espontânea da criança (MARTIN et al.,2016). Já o oferecimento de leite de vaca integral deve ser evitado em crianças menores de 12 meses de vida (KOLETZKO et al., 2013).

Neste sentido, a introdução de chá, água ou quaisquer outros alimentos antes dos 6 meses de idade configura uma prática de interrupção precoce da amamentação exclusiva e representa um risco a saúde para a criança, em especial aquelas prematuras, tendo em vista o retardo no 
desenvolvimento neuromuscular e fisiológico o que implica num maior despreparo desse grupo para receber novos alimentos. Soma-se a isto os riscos oferecidos à saúde por uma introdução alimentar inadequada que aumenta as chances de desenvolver problemas tais como infecções, diarreias, desnutrição, alergias, sobrepeso/obesidade e outras DCNT na adolescência e fase adulta (D’ AURIA et al., 2020; SBP, 2018; LOPES et al., 2018; VICTORA et al., 2016), sendo nos prematuros estes riscos ainda maiores quando a alimentação complementar é iniciada precocemente aumentando as chances de rápido ganho de peso e síndrome metabólica (HEIDEMANN et al., 2019). A alimentação complementar adequada e bem orientada se soma aos benefícios do AME em reduzir riscos em curto e longo prazos, promovendo saúde ao longo da vida. Sendo assim, o presente estudo teve por objetivo investigar a frequência do AME em prematuros ao longo dos 6 primeiros meses de vida, bem como os alimentos mais frequentemente introduzidos às crianças neste período.

\section{MATERIAIS E MÉTODOS}

Trata-se de estudo de coorte com recém-nascidos prematuros nascidos na maternidade de alto risco do Hospital Universitário Professor Alberto Antunes (HUPAA), localizada no município de Maceió, estado de Alagoas, durante os anos de 2016 e 2017. O presente estudo é parte de um projeto maior intitulado "Autoeficácia na amamentação e duração do aleitamento materno: um estudo de coorte com puérperas no pós-parto prematuro" aprovado pelo Comitê de Ética em Pesquisa (CEP) (protocolo $n^{\circ}$ 1.568.544), que contou com uma amostra de 381 crianças prematuras, das quais, 179 estavam em aleitamento materno (AM) e destes 161 em AME na alta hospitalar.

Foram incluídos recém-nascidos prematuros (idade gestacional no parto $<37$ semanas) (WHO, 1961), assistidos na maternidade do hospital, procedentes de Alagoas e que estavam sendo amamentados de forma exclusiva na alta hospitalar. Foram excluídos aqueles recém-nascidos com algum tipo de doença associada que limitasse a alimentação por via oral, bem como aqueles em aleitamento materno misto ou complementado.

Inicialmente, na maternidade do hospital era realizada triagem feita de forma aleatória a partir da análise do prontuário eletrônico individualizado. Na sequência, os entrevistadores, devidamente treinados, deslocavam-se para as enfermarias e, após convite e aceite voluntário para participação no estudo, as mães dos recém-nascidos respondiam a um questionário padronizado. $\mathrm{Na}$ segunda etapa da pesquisa, que aconteceu após a alta hospitalar, as crianças foram acompanhadas por meio de ligações telefônicas realizadas 30, 60, 90, 120, 150 e 180 dias após o nascimento, para avaliação da duração do aleitamento materno. Nesta ocasião, a genitora era questionada seus respectivos filhos eram mantidos em AME ou se já havia sido iniciada a introdução de outros alimentos, configurando uma interrupção precoce do AME. Em caso 
afirmativo para introdução alimentar precoce, também foram investigados os gêneros alimentícios mais frequentemente introduzidos a cada período das ligações.

Os dados foram tabulados e analisados por meio de estatística descritiva com auxílio do programa SPSS version 20.0, e foram expressos por meio de médias com respectivos desviospadrões e/ ou frequência.

\section{RESULTADOS}

Foram avaliado neste estudo 132 prematuros, do total de 161 crianças incluídas no início da coorte, totalizando uma perda de $18,01 \%$ de seguimento da amostra.

Dos 132 prematuros estudados houve uma redução de $67,77 \%$ da frequência de aleitamento materno desde o nascimento, com uma prevalência ao final do período de acompanhamento de 32,23\%. Quando detalhados por período de acompanhamento, as prevalência de AME foram $91,66 \%$ aos 30 dias, $81,81 \%$ aos 60 dias, $67,68 \%$ aos 90 dias, 58,67\% aos 120 dias, $46,28 \%$ aos 150 dias e de $32,23 \%$ aos 180 dias de vida.

$\mathrm{Na}$ Figura 1 podem ser vistas as frequências do AME e de aleitamenro materno misto (AMM) e predominante referidos pelas mães dos prematuros avaliados. Como pode ser observado, à medida que houve redução do AME, como já descrito, verifica-se um aumento do aleitamento materno não exclusivo, onde o aleitamento materno misto foi o mais prevalente na coorte estudada, seguido do aleitamento materno predominante (AMP). Aos 30 dias após o nascimento, 6,06\% das crianças estavam em aleitamento materno misto e $0,75 \%$, em aleitamento materno predominante. Aos 180 dias de vida, 32,57\% das crianças estavam em AMM e 3,78\%, em AMP.

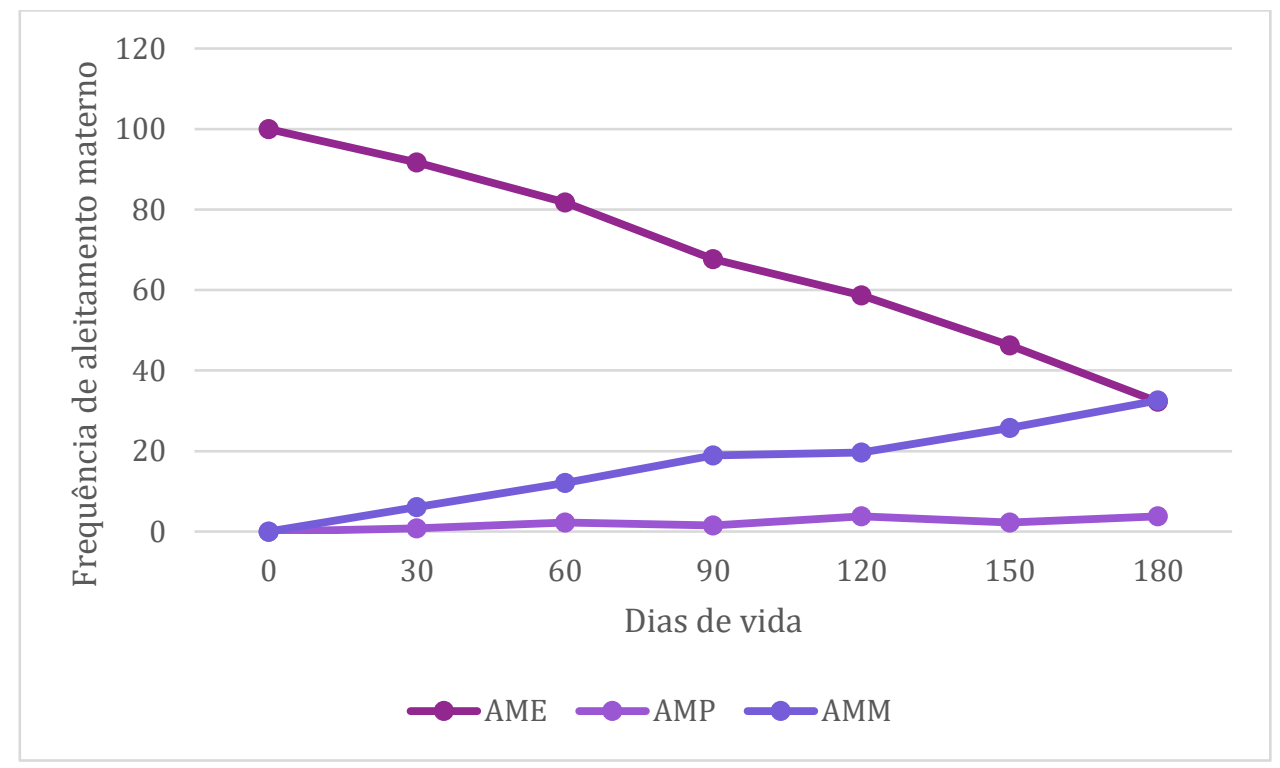


Figura 1. Frequências de aleitamento materno exclusivo (AME), aleitamento materno misto $(\mathrm{AMM})$ e aleitamento materno predominante (AMP) de prematuros ao longo dos 6 primeiros meses de vida. Maceió, Alagoas, 2016/ 2017.

Quanto à introdução alimentar precoce, observa-se nesse estudo, que ainda no primeiro mês de acompanhamento houve introdução de fórmula infantil $(6,06 \%)$, leite de vaca $(1,51 \%)$, farináceos $(1,51 \%)$ e chás $(0,75 \%)$ com aumento do consumo de todos esses alimentos aos 60 dias de acompanhamento. Aos 90 dias, houve introdução de açúcar refinado por uma criança $(0,75 \%)$, aos 120 dias $2(1,51 \%)$ crianças já consumiam suco de frutas, aos 150 dias houve introdução das frutas $(6,06 \%)$, dos legumes e verduras $(2,27 \%)$ e iogurte industrializado $(1,51 \%)$ além do aumento do consumo dos outros alimentos já relatados, e por fim aos 180 dias de acompanhamento, $47(35,6 \%)$ e 48 (36,36\%) estavam em uso de leite de vaca e formula infantil, respectivamente (Tabela 1).

Tabela 1 - Frequência acumulada dos alimentos introduzidos ao longo dos 180 primeiros dias de vida das 132 crianças nascidas prematuras. Maceió, Alagoas, 2016/2017.

\begin{tabular}{|c|c|c|c|c|c|c|}
\hline \multirow{2}{*}{$\begin{array}{l}\text { Alimentos } \\
\text { introduzidos }\end{array}$} & \multicolumn{6}{|c|}{ Tempo (dias) } \\
\hline & 30 & 60 & 90 & 120 & 150 & 180 \\
\hline Fórmula infantil & $8(6,06 \%)$ & $18(13,64 \%)$ & $25(18,94 \%)$ & $25(18,94 \%)$ & $27(20,45 \%)$ & $27(20,45 \%)$ \\
\hline Leite de vaca & $2(1,51 \%)$ & $10(7,58 \%)$ & $21(15,9 \%)$ & $30(22,72 \%)$ & $37(28,03 \%)$ & $47(35,6 \%)$ \\
\hline Farináceo & $2(1,51 \%)$ & $9(6,82 \%)$ & $17(12,89 \%)$ & $28(21,21 \%)$ & $33(25,0 \%)$ & $48(36,36 \%)$ \\
\hline $\begin{array}{l}\text { Iogurte } \\
\text { industrializado }\end{array}$ & 0 & 0 & 0 & 0 & $2(1,51 \%)$ & $3(2,27 \%)$ \\
\hline Frutas & 0 & 0 & 0 & 0 & $8(6,06 \%)$ & $19(14,39 \%)$ \\
\hline Sucos de fruta & 0 & 0 & 0 & $2(1,51 \%)$ & $6(4,54 \%)$ & $20(15,15 \%)$ \\
\hline Legumes e verduras & 0 & 0 & 0 & 0 & $3(2,27 \%)$ & $17(12,88 \%)$ \\
\hline Chás & $1(0,75 \%)$ & $3(2,27 \%)$ & $4(3,03 \%)$ & $4(3,03 \%)$ & $5(3,79 \%)$ & $5(3,79 \%)$ \\
\hline Açúcar refinado & 0 & 0 & $1(0,75 \%)$ & 0 & $2(1,51 \%)$ & $2(1,51 \%)$ \\
\hline
\end{tabular}

\section{DISCUSSÃO}


Nesse estudo, um dos poucos descritos na literatura sobre a introdução alimentar precoce com foco em prematuros, mais de 2/3 $(67,67 \%)$ das crianças avaliadas não estava mais em AME ao longo dos 6 meses (180 dias) de acompanhamento.

O Guia alimentar para crianças brasileiras menores de 2 anos de idade, recentemente revisado, reafirma que o aleitamento materno deve ocorrer de forma exclusiva até os 6 meses, mesmo para aqueles prematuros, não devendo, portanto, ser ofertado água, chás, sucos, fórmulas infantis ou outros tipos de leite. O documento considera a oferta de outros tipos de leite (como o leite de vaca) antes dos 6 meses de vida apenas em casos de impossibilidade de aquisição de fórmulas infantis quando a família se encontra em situação de vulnerabilidade socioeconômica, ou quando há outro motivo que inviabilize a oferta de leite materno (BRASIL, 2019).

Considerando a constatação da redução gradual do AME, um outro estudo desenvolvido em Fortaleza-CE com recém-nascidos a termo, encontrou uma menor prevalência do AME $(6,5 \%)$ ao fim dos 6 meses de acompanhamento, desde o parto, resultado bem inferior ao encontrado no presente estudo (FERREIRA et al., 2018). Em outro estudo desenvolvido no estado da Pensilvânia, nos Estados Unidos (HACKMAN et al., 2016), que avaliou a prevalência da amamentação em prematuros, verificou-se uma menor prevalência de $\operatorname{AME~}(63,8 \%)$ ao final do $1^{\circ}$ mês de pós-parto, frequência bem inferior ao observado no presente trabalho, que foi de $91,66 \%$.

De forma geral, os prematuros são mais propensos a apresentarem desfechos adversos à saúde, haja vista que fisiologicamente o organismo imaturo necessita se adaptar a processos metabólicos que não estava preparado. Assim, as evidências mostram que o AME é uma das estratégias com grande potencial de proteção contra doença por ser um alimento completo que irá fornecer componentes nutricionais em quantidades ideiais para essas crianças (CERASANI et al., 2020).

As crianças de 0 a 6 meses de vida são fortemente orientadas a receber AME, a menos que tenham orientações especificas para a oferta de outros tipos de alimentos. Assim, crianças que não recebem leite materno de forma exclusiva muitas vezes é ofertado o AMP, ou seja, quando a criança além do leite materno recebe água ou bebidas a base água ou AMM ou parcial, onde a criança recebe leite materno e outros tipos de leite (BRASIL, 2015).

Estudo realizado na Indonésia, na Ásia, verificou que lactentes em AMM durante os 6 primeiros meses de vida tiveram um risco de 14 vezes maior de apresentarem infecções respiratórias agudas (JANSEN et al., 2020). Ainda, a introdução precoce de outros alimentos que não seja o leite materno oferece um risco maior de contaminação por patógenos, como contaminantes em mamadeiras, bicos e/ou em água (QUIGLEY et al., 2016). Portanto, recomenda-se a introdução de novos alimentos apenas após os 6 meses de vida da criança (BRASIL, 2015). 
No presente estudo, observou-se que as crianças que não estavam mais em AME receberam como introdução precoce fórmula infantil, leite de vaca, farináceos, chás, suco de frutas, frutas, legumes, verduras e iogurte industrializado. Há informações importantes na literatura quanto a exposição precoce de novos alimentos na rotina da criança que não seja o leite materno e, em especial para os prematuros, esse tipo de conduta pode ter como fator associado a idade e escolaridade materna, idade gestacional de nascimento da criança, número de gestações anteriores e a renda familiar (HOFSTATTER et al., 2021).

Um estudo brasileiro desenvolvido com dados de registro alimentar de menores de 6 meses de vida provenientes do Sisvan Web, apontou a água ou chás $(28,9 \%)$ e as fórmulas infantis $(25,00 \%)$ como os alimentos mais introduzidos precocemente, sendo as fórmulas associadas a maior déficit ponderal quando comparado aos prematuros amamentados exclusivamente. Nesse trabalho, os autores identificaram ainda a introdução precoce de frutas e sucos de frutas $(11,5 \%$ e 13,00\%, respectivamente) (GONÇALVES et al., 2019).

Ainda, estudo realizado no Rio de Janeiro-RJ com 108 crianças prematuras, objetivando avaliar a qualidade da dieta sobre o estado nutricional de prematuros, observou uma maior frequência do uso exclusivo de fórmula infantil (47,3\%), seguido de aleitamento misto $(34,5 \%)$ e AME $(16,7 \%)$ na alta hospitalar. Ao longo do acompanhamento os autores identificaram o abandono no uso de fórmula em detrimento do consumo de leite de vaca, que alcançou 57,4\% aos 12 meses de idade corrigida da criança, além de uma introdução alimentar precoce em 77,6\% dos prematuros entre 4 e 6 meses de idade corrigida (RIBAS et al., 2020).

Um estudo australiano determinou a primeira idade de introdução precoce de alimentos sólidos em prematuros em comparação com recém-nascidos a termo. Os autores demonstraram uma maior frequência de introdução alimentar precoce em prematuros $(83,00 \%)$, ocorrida por volta das 21 semanas de idade cronológicas e 17 semanas de idade corrigida, além de demonstrarem uma maior frequência na introdução de farináceo como primeiro alimento sólido (68,00\%) (CLEARY et al., 2019).

Muito se discute na literatura sobre os desfechos clínicos desfavoráveis da introdução alimentar precoce em curto e longo prazos. Enquanto algumas publicações tratam como insuficiente ou imprecisos os dados disponíveis que relacionam o ganho ponderal excessivo e risco de alergias com o momento da introdução alimentar em prematuros, outras confirmam a ocorrência destes desfechos, além de considerar o risco de outras complicações como o diabetes tipo 2, enterocolite necrotizante, aumento nas taxas de sepse e maior tempo em nutrição parenteral. Variações na microbiota intestinal, introdução precoce do leite de vaca, o tipo e o uso incorreto de fórmulas infantis em prematuros são alguns fatores que podem mediar o desenvolvimento de tais desfechos pela exposição precoce as proteínas do leite de vaca que, por sua vez, apresentam potencial alergênico (LIOTTO et al., 2020; HAY, HENDRICKSON, 2016; MENNELLA et al., 2018; BRION et al., 2020; BURGE et al., 2018). 
Alguns aspectos culturais que envolvem a amamentação podem explicar, ao menos em parte, a introdução precoce de alimentos sólidos. A presença do choro pode indicar algum incomodo para o lactente, porém muitas vezes é interpretado como fome. Nesse raciocínio o leite materno é equivocada e comumente citado por muitos como sendo "fraco", levando a adição de farináceos para engrossar leites, fórmulas infantis e papas como estratégia para aumento da saciedade no lactente (BRASIL, 2019).No contexto do Sistema Único de Saúde (SUS) se reconhece uma série de vulnerabilidades sociais que tornam ainda mais delicada as condições para introdução alimentar adequada num público já marcado por uma série de vulnerabilidades biológicas decorrentes da prematuridade, corroborando com a necessidade de mais trabalhos como este. Desta forma, a partir da compreensão desta realidade, é possível que sejam traçadas estratégias de enfrentamento de tal problema com foco na promoção do aleitamento materno nos diferentes níveis de atenção à saúde.

Como limitações do presente trabalho pode ser apontado as dificuldades em contactar as puérperas via ligação telefônica, a partir dos contatos fornecidos, além do fato de não conseguirmos de forma mais detalhada as informações sobre os alimentos por hora introduzidos à alimentação das crianças, ao longo do período de acompanhamento.

\section{CONCLUSÃO}

No presente trabalho, foi observada uma redução na prevalência AME em detrimento ao aumento da frequência de introdução precoce de alimentos como fórmulas infantis, leite de vaca, farináceios, chás, suco de frutas, frutas, legumes, verduras e iogurte industrializado.

Diante disto, aponta-se aqui a necessidade de estratégias que visam a manutenção adequada da amamentação durante os 6 primeiros meses de vida, especialmente nos prematuros, momento no qual a oferta do leite materno deve ser exclusiva. Havendo impossibilidade do AME, puérperas devem ser devidamente orientadas e acompanhadas por profissionais adequadamente capacitados, atuantes na rede básica de saúde, afim de que a introdução alimentar se dê de forma orientada e adequada. Este aspecto se faz especialmente importante, uma vez que se reconhece as fragilidades próprias da prematuridade e os riscos que esta condição acarreta aos conceptos e o papel da alimentação em reduzir os impactos de tais riscos sobre o estado de saúde em curto e longo prazos.

\section{RFERÊNCIAS}

BRAID, S; HARVEY, E.M.; BERNSTEIN, J.; MATOBA, N. Early Introduction of Complementary Foods in Preterm Infants. Journal of Pediatric Gastroenterology and Nutrition, v.60, n.6, p.811-18, 2015. 
BRASIL. Ministério da Saúde. Secretaria de Atenção à Saúde. Departamento de Atenção Básica. Saúde da criança: aleitamento materno e alimentação complementar. Cadernos de Saúde. n.23. 2ª ed. Brasília: Ministério da Saúde, 2015.

BRASIL. Ministério da Saúde. Secretaria de Atenção Primária à Saúde. Departamento de Promoção à Saúde. Guia Alimentar para crianças brasileiras menores de 2 anos / Ministério da Saúde / Secretaria de Atenção Primária à Saúde / Departamento de Promoção à Saúde. Brasília: Ministério da Saúde, 2019.

BRION, L.P et al. Association of age of initiation and type of complementary foods with body mass index and weight-for-length at 12 months of age in preterm infants. Journal of Perinatalogy, v.40, p.1394-1404, 2020.

BURGE, D.M.; DREWETT, M.; HALL, N.J. The temporal relationship between exposure to bovine milk products and surgical NEC in preterm infants. Infant, v.14, n.2, p.58-61, 2018.

CERASANI, J. et al. Human milk feeding and preterm infants' growth and body composition: A literature review. Nutrients, v. 12, n. 4, p. 1155, 2020.

CLEARY, J.; DALTON, S.M.C.; HARMAN, A.; WRIGHT, I.M. Current practice in the introduction of solid foods for preterm infants. Public Health Nutrition, v.23, n.1, p. $94-101,2019$.

D'AURIA, E. et al. Complementary feeding: pitfalls for health outcomes. International journal of environmental research and public health, v. 17, n. 21, p. 7931, 2020.

FERREIRA, H.L.O.C.; OLIVEIRA, M.F.D.; BERNARDO, E.B.R.; ALMEIDA, P.C.D.; AQUINO, P.D.S.; PINHEIRO, A.K.B. Fatores Associados à adesão ao aleitamento materno exclusivo. Ciência \& Saúde Coletiva, v.23, p. 683-690, 2018.

GONÇALVES, V.S.S.; SILVA, A.S.; ANDRADE, R.C.S.; SPANIOL, A.M.; NILSON, E.A.F.; MOURA, I.F. Marcadores de consumo alimentar e baixo peso e crianças menores de 6 meses acompanhadas no Sistema de Vigilância Alimentar e Nutricional, Brasil, 2015. Epidemiol. Serv. Saúde, v.28, n.2, p. e2018358, 2019.

HACKMAN, N.M.; ALLIGOOD-PERCOCO, N.; MARTIN, A.; ZHU, J.; KJERULFF, K.H. Reduced breastfeeding rates in firstborn late preterm and early term infants. Breastfeeding Medicine, v.11, n.3, p.119-125, 2016.

HAY JR, W.W.; HENDRICKSON, K.C. Preterm formula use in the preterm very low birth weight infant. Seminars in Fetal \& Neonatal Medicine, v.22, n.1, p. 15-22, 2016.

HEIDEMANN, L.A.; PROCIANOY, R.S.; SILVEIRA, R.C. Prevalence of metabolic syndrome-like at 2 years of corrected age in very low birth weight preterm infants and associated factors. Jornal de Pediatria, v.95, n.3, p. 291-297, 2019.

HOFSTÄTTER, E. et al. Introduction and feeding practices of solid food in preterm infants born in Salzburg!. BMC pediatrics, v. 21, n. 1, p. 1-11, 2021. 
JANSEN, S. et al. Mothers' knowledge of breastfeeding and infant feeding types affect acute respiratory infections. Journal of Preventive Medicine and Hygiene, v. 61, n. 3, p. E401, 2020.

$\mathrm{KOH}$, Kanghyock. Maternal breastfeeding and children's cognitive development. Social Science \& Medicine, v. 187, p. 101-108, 2017.

KOLETZKO, B. et al. European Childhood Obesity trial Study. Early influences of nutrition on postnatal growth. Nestle Nutr. Inst. Workshop Ser, v. 71, p. 11-27, 2013.

LANCET BREASTFEEDING SERIES GROUP. Breastfeeding in the 21st century: epidemiology, mechanisms, and lifelong effect. Lancet 2016; 387: 475-90.

LIMA, A.P.C.; NASCIMENTO, D.S.; MARTINS, M.M.F. A prática do aleitamento materno e os fatores que levam ao desmame precoce: uma revisão integrativa. J. Health Biol Sci., v.6, n.2, p.189-19, 2018.

LIOTTO, N. et al. Complementary Feeding in Preterm Infants: A Systematic Review. Nutrients, v.12, n.6, p. 1843, 2020.

LOPES, W.C. et al. Alimentação de crianças nos primeiros 2 anos de vida. Rev Paul Pediatr., v.36, n.2, p. 164-70, 2018.

MARTIN, C.R.; LING, P.R; BLACKBURN, G.L. Review of infant feeding: key features of breast milk and infant formula. Nutrients, v. 8, n. 5, p. 279, 2016. MENNELLA, J. et al. Type of infant formula increases earrly weight gain and impacts energy balance: a randomized controlled trial. The American Journal of Clinical Nutrition, v. 108, n.5, p.1015-1025, 2018.

OLIVEIRA, I.B.B.; LEAL, L.P.; CORIOLANO-MARINUS, M.W.L.; SANTOS, A.H.S.; HORTA, B.L.; PONTES, C.M. Meta-analysis of the effectiveness of educational interventions for breastfeeding promotion directed to the woman and her social network. Journal of advanced nursing, v.73, n.2, p. 323-335, 2017.

QUIGLEY, M. A. et al. Exclusive breastfeeding duration and infant infection. European journal of clinical nutrition, v. 70, n. 12, p. 1420-1427, 2016.

RIBAS, A.S.; RODRIGUES, M.C.C.; MOCELLIN, M.C.; MARQUES, E.S.; ROSA, G.P.C.; MAGANHA, C.R. Quality of complementary feeding and its effect on nutritional status in preterm infants: a cross-sectional study. Journal of Human Nutrition and Dietetics, v.34, n.1, p. 3-12, 2020.

SOCIEDADE BRASILEIRA DE PEDIATRIA. Departamento de Nutrologia. Manual de Alimentação da infância à adolescência. $4^{a}$ edição. São Paulo: SBP, 2018.

VICTORA, C.G. et al. Breastfeeding in the 21st century: epidemiology, mechanisms, and lifelong effect. The Lancet, v. 387, n. 10017, p. 475-90, 2016.

WERE, Fred N.; LIFSCHITZ, Carlos. Complementary feeding: Beyond nutrition. Annals of Nutrition and Metabolism, v. 73, p. 20-25, 2018. 
WILSON, E. et al. Room for improvement in breast milk feeding after very preterm birth in Europe: Results from the EPICE cohort. Maternal \& Child Nutrition, v.14, n.1, p. e12485, 2018.

WORLD HEALTH ORGANIZATION. Health aspects of low birth weight. Tech Rep Series. Geneva 1961, 217.

Recebido em: 08/01/2022

Aprovado em: 10/02/2022

Publicado em: 14/02/2022 